ACCEPTED MANUSCRIPT

\title{
The physics of thermal light second-order interference beyond coherence
}

To cite this article before publication: Vincenzo Tamma et al 2018 Phys. Scr. in press https://doi.org/10.1088/1402-4896/aae71C

\section{Manuscript version: Accepted Manuscript}

Accepted Manuscript is "the version of the article accepted for publication including all changes made as a result of the peer review process, and which may also include the addition to the article by IOP Publishing of a header, an article ID, a cover sheet and/or an 'Accepted Manuscript' watermark, but excluding any other editing, typesetting or other changes made by IOP Publishing and/or its licensors"

This Accepted Manuscript is @ 2018 IOP Publishing Ltd.

During the embargo period (the 12 month period from the publication of the Version of Record of this article), the Accepted Manuscript is fully protected by copyright and cannot be reused or reposted elsewhere.

As the Version of Record of this article is going to be / has been published on a subscription basis, this Accepted Manuscript is available for reuse under a CC BY-NC-ND 3.0 licence after the 12 month embargo period.

After the embargo period, everyone is permitted to use copy and redistribute this article for non-commercial purposes only, provided that they adhere to all the terms of the licence https://creativecommons.org/licences/by-nc-nd/3.0

Although reasonable endeavours have been taken to obtain all necessary permissions from third parties to include their copyrighted content within this article, their full citation and copyright line may not be present in this Accepted Manuscript version. Before using any content from this article, please refer to the Version of Record on IOPscience once published for full citation and copyright details, as permissions will likely be required. All third party content is fully copyright protected, unless specifically stated otherwise in the figure caption in the Version of Record.

View the article online for updates and enhancements. 


\title{
1. Introduction and motivation
}

The discover of the Hanbury Brown and Twiss (HBT) effect [2,3] in 1956 triggered an intense debate about the physics behind this effect and even its correctness. To quote Hanbury Brown, the reason behind a lot of this criticism was that "many physicists had failed to grasp how profoundly mysterious light really is". However, as often happens in science for a new phenomenon which is initially not well understood and received, the HBT effect paved the way to the development of an entire new field, the field of quantum optics $[4,5,6,7,8]$. One can consider this discovery as a precursor of fundamental studies and experiments based on multi-photon interference and correlations $[9,10,11,12,13,14,15,16,17,18,1,19]$, with intriguing applications in imaging $[20,21,22,23,24,25,26,27,28,29,30]$, quantum information processing [31, 32, 33, 34, 35, 36], and metrology [37, 38, 39, 40, 41, 42, 43, 44].

In the temporal domain [2], the HBT effect consists of beam splitting a thermal beam and measuring the correlation in time between the photon intensities at the beam splitter output ports (Fig. 1(a)). Two-photon interference is observed for a detection time delay small compared to the coherence time of the source. Indeed, twofold detection events have twice the chance to occur at equal detection times than with a

\section{The physics of thermal light second-order interference beyond coherence}

\begin{abstract}
A novel thermal light interferometer was recently introduced in Ref. [1]. Here, two classically correlated beams, obtained by beam splitting a thermal Remarkably, second-order interference between the long and the short paths in the two interferometers was predicted independently of how far, in principle, the length difference between the long and short paths is beyond the coherence length of the source. This phenomenon seems to contradict our common understanding of secondorder coherence. We provide here a simple description of the physics underlying this effect in terms of two-photon interference.

Vincenzo Tamma 3QL, UK Sosmology \& Gravitation, University of Portsmouth, Portsmouth PO1

E-mail: vincenzo.tamma@port.ac.uk

June 2018
\end{abstract}




\section{The physics of thermal light second-order interference beyond coherence}

(a)

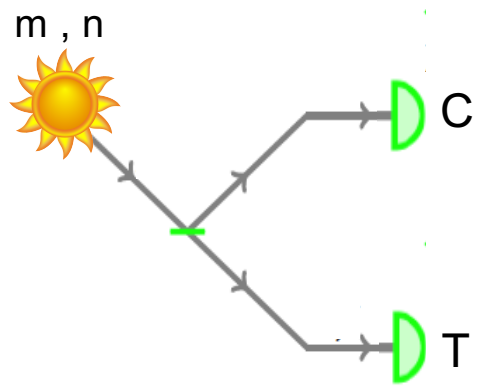

(b)
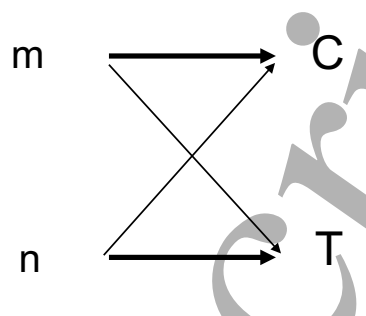

Figure 1: (a) Hanbury Brown and Twiss (HBT) interferometer: The classically correlated beams obtained by beam splitting the light emitted by a thermal source are measured by performing joint detections at the two output ports of the balanced beam splitter. (b) Two-photon interference is observed at detection time delays small compared with the coherence time of the source. This corresponds to the interference of two two-photon detection amplitudes corresponding to the two possible indistinguishable paths (depicted with bold and thin arrows, respectively) two photons $\mathrm{m}$ and $\mathrm{n}$ can undertake to trigger a joint detection.

time delay much larger than the coherence time. This effect arises from the interference between the two possible indistinguishable pairs of paths that two detected photons may have undertaken from the source to the two detectors (Fig. 1(b)). We emphasize that, since these two pairs of path completely overlap in space, the HBT effect is not sensitive to any relative phase delay. Indeed, no sinusoidal fringes can be observed in standard HBT experiments.

Recently, was proposed a novel interferometric scheme, depicted in Fig. 2(a), where, differently from the standard HBT scheme, two Mach-Zehnder interferometers are introduced at the two output ports of the beam splitter before a correlation measurement at approximately equal detection times is performed [1]. Furthermore, the difference between the long (dashed red) paths $L=L_{C}, L_{T}$ and the short (dotted blue) paths $S=S_{C}, S_{T}$ in each Mach-Zehnder interferometer is assumed to be larger than the coherence length of the source, while the differences in length between paths of the same type, $L_{C}-L_{T}$ and $S_{C}-S_{T}$, are negligible with respect to the coherence length.

This interferometer has some similarities with the famous Franson interferometer [13], which however differs in two fundamental aspects:

- a source of two classically correlated beams obtained by beam splitting a thermal light beam is replaced in the Franson interferometer by a source of two photons entangled in energy and time;

- the time delay between the long and short paths in each Mach-Zehnder interferometer is required to be within the second-order coherence time of the twophoton source, which is usually given by the coherence time of the pump laser which generates the entangled pair of photons when interacting with an SPDC crystal.

These two conditions ensure the emission of two entangled photons simultaneously 
(a)

(b)
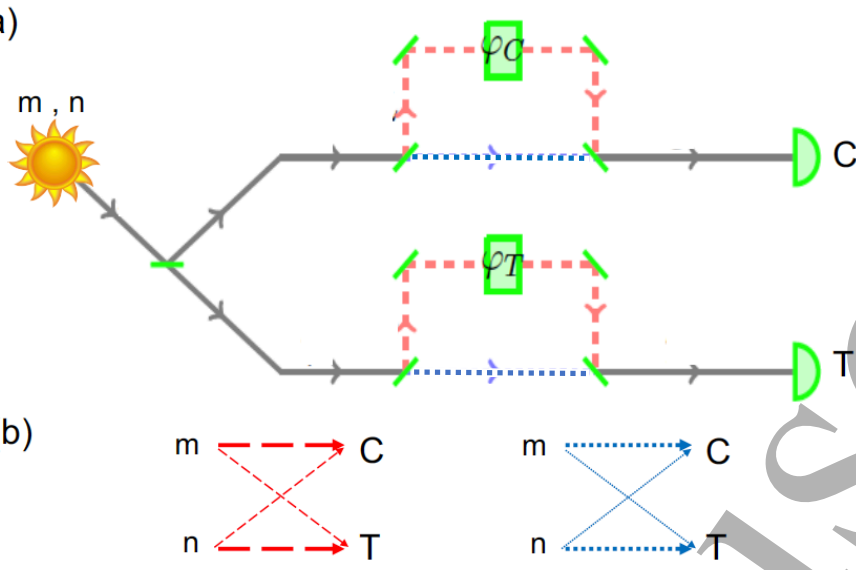

(c)
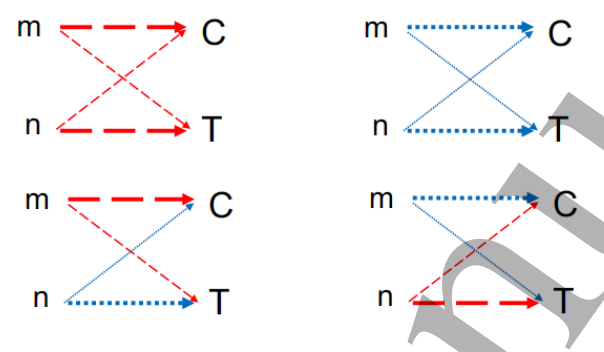

Figure 2: (a) Thermal light interferometer introduced in Ref. [1]: A source of classically correlated beams as in the HBT interferometer in Fig. 1(a) is used. However, correlated measurements are not performed directly at the two output ports of the beam splitter but after the light propagates through two unbalanced Mach-Zehnder interferometers with relative phases $\varphi_{C}$ and $\varphi_{T}$, respectively. At detection time delays small compared with the coherence time of the source, second-order interference is observed between the pair of long (dashed red) paths $L=L_{C}, L_{T}$ and the pair of short (dotted blue) paths $S=S_{C}, S_{T}$, which length difference $L-S$ is beyond the coherence length of the source. This interference is a result of the collective contribution of all the possible ways twophoton interference can arise between two pairs of two-photon paths (indicated by bold and thin arrows, respectively) undertaken by any given pair $(m, n)$ of detected photons emitted by the thermal source: (b) two-photon interference between the $\left(L_{C}, L_{T}\right)$ and $\left(L_{T}, L_{C}\right)$ (or $\left(S_{C}, S_{T}\right)$ and $\left(S_{T}, S_{C}\right)$ ) pairs of paths, which is phase-independent as in standard HBT experiments; (c) two-photon interference between the $\left(L_{C}, S_{T}\right)$ and $\left(L_{T}, S_{C}\right)$ (or $\left(S_{C}, L_{T}\right)$ and $\left(S_{T}, L_{C}\right)$ ) pairs of paths, which is instead sensitive to the phase difference $\varphi_{C}-\varphi_{T}$.

but with a "quantum uncertainty" in time (second-order coherence time) larger than the time delay $(L-S) / c$ due to the unbalance between the long and short paths in each Mach-Zehnder interferometer, even if the usual first order coherence length of each single photon is much smaller than the path length difference $L-S$. This is at the very heart of the observation of the well known two-photon Franson interference between the two pairs $\left(L_{C}, L_{T}\right)$ and $\left(S_{C}, S_{T}\right)$ of two-photon paths.

Can second order interference between the path configurations $\left(L_{C}, L_{T}\right)$ and $\left(S_{C}, S_{T}\right)$ be also observed in the thermal light interferometer in Fig. 2(a) where the path length difference $|L-S| / c$ in each Mach-Zehnder interferometer is beyond the coherence 


\section{The physics of thermal light second-order interference beyond coherence}

length of the thermal source? Does it matter here how far beyond? Interestingly, as shown in Ref. [1], the answer is yes and it does not matter, in principle, how far beyond. This result is highly counter-intuitive since, differently from the Franson interferometer, no energy-time entanglement occurs to justify any two-path indistinguishability in terms of second-order coherence length. How does such two-path indistinguishability arise? How can we reconcile it with our common understanding of second-order coherence?

Even more interestingly, it was predicted that this second-order interference phenomenon with thermal light, differently from the standard HBT effect, could manifest itself in sinusoidal fringes as a function of the difference $\varphi_{C}-\varphi_{T}$ between the phase delays in the two Mach-Zehnder interferometer [1]. This differs from the Franson interferometer which is sensitive to the sum $\varphi_{C} 4 \varphi_{T}$ of the interferometer relative phases.

But what is the physics behind the observation of these sinusoidal fringes? And why such a fundamental difference with respect to the Franson interferometer?

All these questions have puzzled different scientists who were reluctant in believing that this interference effect was even correct when it was first predicted and no experimental realizations were yet performed. Fortunately, two main features were of substantial help in experimentally verifying this phenomenon: 1) a thermal source can be easily simulated in a laboratory by using laser light impinging on a fast rotating ground glass [45]; 2) given a coherence time for a thermal source which can range from the order of $n s$ to $\mu s$, the calibration of the interferometric paths and of the detection times can be easily achieved. Furthermore, the same effect can be realised also in the spatial domain, by substituting the two Mach-Zehnder interferometers with two double slits and performing spatially correlated measurements at the output, as proposed in Ref. [40]. Indeed, it did not take too long until experimental demonstrations of this effect were first reported both in the temporal and spatial domain in Refs. [19, 41], respectively. Furthermore, as predicted in Ref. [1], interference fringes as a function of the phase difference $\varphi_{C}-\varphi_{T}$ have been observed with almost constant visibility at the increasing of the path length difference $L-S$ even if of the order of $680 \mathrm{~m}$ beyond the coherence length of the source [19]. This phenonenon therefore paves the way to applications in high precision metrology and sensing, including the characterization of remote objects as demonstrated first theoretically in Ref. [40] and than experimentally in Ref. [41].

In addition, the genuineness of the correlations between the long and the short paths in the observed second order interference is testified by the possibility to exploit them to simulate quantum logic gates in absence of entanglement [1]. Indeed, the simulation of a CNOT gate by using this interference effect, first theoretically proposed in the temporal domain in Ref. [1] and extended to the spatial domain in Ref. [40], was experimentally realisedin Ref. [46]. This can lead to potential applications in the development of novel optical algorithms with thermal light [47, 48, 49, 50, 51].

In this paper, we provide a deeper understanding of the physics behind this interference phenomenon in terms of interference of "two-photon detection amplitudes" 


\section{The physics of thermal light second-order interference beyond coherence}

[9, 10]. We will demonstrate how, differently from standard HBT experiments, interference can actually occur in a phase-dependent manner between two-photon detection amplitudes corresponding to the propagation of a pair of detected photons $(m, n)$ from the source to the detectors $d=, C, T$ through two possible pairs $\left(L_{C}, S_{T}\right)$ and $\left(L_{T}, S_{C}\right)$ of two different types of paths $L$ and $S$ (Fig. 2(c)). Further, standard HBT interfering amplitudes also arise from the propagation through pairs of the same type of path (either $\left(L_{C}, L_{T}\right)$ interfering with $\left(L_{T}, L_{C}\right)$ or $\left(S_{C}, S_{T}\right)$ interfering with $\left(S_{T}, S_{C}\right)$ (Fig. 2(b)).

It is than the combination of all these interference contributions from all the possible pairs of photons emitted by the source that leads to the observed "collective" interference between the pair $\left(L_{C}, L_{T}\right)$ of long paths and the pair $\left(S_{C}, S_{T}\right)$ of short paths. Evidently, this does not mean that each pair $(m, n)$ of two detected/photons have either taken the $\left(L_{C}, L_{T}\right)$ or the $\left(S_{C}, S_{T}\right)$ paths in an indistinguishable manner. Indeed, the correlated paths $\left(S_{C}, S_{T}\right)$ and $\left(L_{C}, L_{T}\right)$ cannot be interpreted as two-photon paths. On the other hand, their interference is a result of the non trivial collective contribution of all the possible pairs $(m, n)$ of detected photons associated with different interfering two-photon detection amplitudes within the statistics of the thermal source (Figs. 2(b) and 2(c)).

In Section 2, we will first analyze this interference phenomenon by using the standard description of thermal light in terms of the Glauber-Sudarshan probability distribution (Eq. (2)). In Section 3, we will give a detailed analysis of the fundamental two-photon interference physics underlying this effect. We will finally conclude the paper with conclusions and perspectives in Section 4.

\section{Thermal light interference "beyond coherence"}

We consider the interferometer in Fig. 1(a), with the input thermal state described by $[5,8]$

$$
\hat{\rho}_{\text {ther }}=\int\left[\prod_{\omega} \mathrm{d}^{2} \alpha_{\omega}\right] P_{\hat{\rho}_{\text {ther }}}\left(\left\{\alpha_{\omega}\right\}\right) \bigotimes_{\omega}\left|\alpha_{\omega}\right\rangle\left\langle\alpha_{\omega}\right|,
$$

with the Glauber-Sudarshan probability distribution [9, 52]

$$
P_{\hat{\rho}_{\text {ther }}}\left(\left\{\alpha_{\omega}\right\}\right)=\prod_{\omega} \frac{1}{\pi \bar{n}_{\omega}} \exp \left(-\frac{\left|\alpha_{\omega}\right|^{2}}{\bar{n}_{\omega}}\right)
$$

and the average photon number [5]

$$
\bar{n}_{\omega}=\bar{r} \frac{1}{\sqrt{2 \pi} \Delta \omega} \exp \left\{-\frac{\left(\omega-\omega_{0}\right)^{2}}{2 \Delta \omega^{2}}\right\}
$$

at frequency $\omega$, with the mean photon rate $\bar{r}$, average frequency $\omega_{0}$ and spectral width $\Delta \omega$. 
The physics of thermal light second-order interference beyond coherence

At the interferometer output the intensity fluctuations

$$
\Delta I_{d}\left(t_{d}\right)=I_{d}\left(t_{d}\right)-\left\langle I_{d}\left(t_{d}\right)\right\rangle
$$

at the detection time $t_{d}$ around the mean value $\left\langle I_{d}\left(t_{d}\right)\right\rangle$, at each detector $d=C, T$, can be measured by using single-photon detectors with integration time $\delta t \ll 1 / \Delta \omega[53,54]$. One can than evaluate the correlation in the intensity fluctuations at the two output ports $[53,54,5]$

$$
\left\langle\Delta I_{C} \Delta I_{T}\right\rangle=\left\langle I_{C} I_{T}\right\rangle-\left\langle I_{C}\right\rangle\left\langle I_{T}\right\rangle
$$

by subtracting from the correlation in the intensities [5]

$$
\left\langle I_{C}\left(t_{C}\right) I_{T}\left(t_{T}\right)\right\rangle \propto G^{(2)}\left(t_{C}, t_{T}\right)=\operatorname{tr}\left[\hat{\rho}_{\text {ther }} \hat{E}_{C}^{(-)}\left(t_{C}\right) \hat{E}_{T}^{(-)}\left(t_{T}\right) \hat{E}_{C}^{(+)}\left(t_{C}\right) \hat{E}_{T}^{(+)}\left(t_{T}\right)\right],
$$

in terms of the field operators $\hat{E}_{d}^{(+)}\left(t_{d}\right)$ and their Hermitian conjugate $\hat{E}_{d}^{(-)}\left(t_{d}\right)$ at the detectors $d=C, T$, the background term [1]

$$
\left\langle I_{C}\left(t_{C}\right)\right\rangle\left\langle I_{T}\left(t_{T}\right)\right\rangle \propto G^{(1)}\left(t_{C}, t_{C}\right) G^{(1)}\left(t_{T}, t_{T}\right)=2 a^{2} \bar{r}^{2},
$$

associated with the product of the intensities

$$
G^{(1)}\left(t_{d}, t_{d}\right):=\operatorname{tr}\left[\hat{\rho}_{t h e r} \hat{E}_{d}^{(-)}\left(t_{d}\right) \hat{E}_{d}^{(+)}\left(t_{d}\right)\right]
$$

where $a$ is a constant.

At approximately equally detection/times with respect to the coherence time $1 / \Delta \omega$ of the source,

and for path delays

$$
\left|t_{C}-t_{T}\right| \ll 1 / \Delta \omega
$$

$$
\left|L_{C}-L_{T}\right| / c,\left|S_{C}-S_{T}\right| / c \ll 1 / \Delta \omega, \quad\left|L_{T}-S_{T}\right| / c,\left|L_{C}-S_{C}\right| / c \gg 1 / \Delta \omega
$$

the expression in Eq. (4) becomes [1]

$$
\begin{aligned}
\left\langle\Delta I_{C} \Delta I_{T}\right\rangle & \propto\left|G^{\left(L_{C}, L_{T}\right)}\left(t_{C}, t_{T}\right)+G^{\left(S_{C}, S_{T}\right)}\left(t_{C}, t_{T}\right)\right|^{2} \\
& =2 a^{2} \bar{r}^{2}\left(1+\cos \left(\varphi_{C}-\varphi_{T}\right)\right)
\end{aligned}
$$

Here, the interference between the two-path contributions [1]

$$
G^{\left(l_{C}, l_{T}\right)}\left(t_{C}, t_{T}\right)=\mathrm{i} a \bar{r} \mathrm{e}^{\mathrm{i} \omega_{0}\left[t_{C}-t_{T}\right]} \mathrm{e}^{-\mathrm{i} \omega_{0}\left[l_{C}-l_{T}\right] / c} \mathrm{e}^{\left[l_{C}-l_{T}\right]^{2} \Delta \omega^{2} / 2 c^{2}} \mathrm{e}^{-\left[t_{C}-t_{T}\right]^{2} \Delta \omega^{2} / 2},
$$

with $\left(l_{C}, l_{T}\right)=\left(L_{C}, L_{T}\right),\left(S_{C}, S_{T}\right)$, leads to a sinusoidal dependence on the difference $\varphi_{C}-\varphi_{T}$ between the relative phases $\varphi_{d}=\omega_{0}\left(L_{d}-S_{d}\right) / c$, with $d=C, T$, in the two MachZehnder interferometers. These sinusoidal oscillations can be measured independently 


\section{The physics of thermal light second-order interference beyond coherence}

of how much the difference in length between the L-type paths and the S-type path is beyond the coherence length of the source, since each interfering contribution in Eq. (10) depends only on the relative distance between paths of the same type. Indeed, the other interfering terms $G^{\left(S_{C}, L_{T}\right)}\left(t_{C}, t_{T}\right)$ and $G^{\left(L_{C}, S_{T}\right)}\left(t_{C}, t_{T}\right)$ from path of different types give a negligible contributions to Eq. (10) in the conditions in Eq. (9).

We point out that the phase difference $\varphi_{C}-\varphi_{T}=\omega_{0}\left[\left(L_{C}-L_{T}\right)-\left(S_{C}>S_{T}\right)\right] / c$ can be tuned by varying the difference in length between paths of the same type $\left(0 \leq \omega_{0}\left(l_{C}-l_{T}\right) / c \leq 1\right)$, within the coherence length of the source as in Eq. (9). This was obtained, for example, experimentally in Ref. [19] by using a thermal source of coherence length of about $120 \mathrm{~m}$ and central wavelength of $780 \mathrm{~nm}$ by varying the differences in the path lengths with piezoelectric actuators at the rate of $63 \mathrm{~nm} / \mathrm{s}$. We also emphasize that interference fringes can be observed also by measuring directly the correlation in the photon numbers in Eq. (5) but with a visibility of $1 / 3$ due to the additional background term in Eq. (6).

How can we interpret these second-order interference fringes in terms of two-photon interference?

\section{Two-photon interference physics}

We describe here the observed interference effect in terms of interference of two-photon detection amplitudes [9, 10]. Toward this goal, it useful to mimic the thermal field emitted from the point-like source by a very large number of subfields $m$ with frequency $\omega_{m}$, which state can be written in the coherent representation as [7]

$$
|\Psi\rangle \doteq \prod_{m}\left|\alpha_{m}\left(\omega_{m}\right)\right\rangle,
$$

where $\left|\alpha_{m}\left(\omega_{m}\right)\right\rangle$ is an eigenstate of the annihilation operator $\hat{a}_{m}\left(\omega_{m}\right)$ with eigenvalues $\alpha_{m}\left(\omega_{m}\right)$ which contain a real-positive amplitude $a_{m}\left(\omega_{m}\right)$ and a random phase $\varphi_{m}\left(\omega_{m}\right)$ arising from the thermal nature of the subfields emitted by the source.

We can then evaluate the correlation in the intensity fluctuations

$$
\begin{aligned}
\left\langle\Delta I_{C}\left(t_{C}\right) \Delta I_{T}\left(t_{T}\right)\right\rangle & \propto\left\langle\left\langle\Psi\left|\hat{E}^{(-)}\left(t_{C}\right) \hat{E}^{(-)}\left(t_{T}\right) \hat{E}^{(+)}\left(t_{C}\right) \hat{E}^{(+)}\left(t_{T}\right)\right| \Psi\right\rangle\right\rangle_{E s} \\
& -\left\langle\left\langle\Psi\left|\hat{E}^{(-)}\left(t_{C}\right) \hat{E}^{(+)}\left(t_{C}\right)\right| \Psi\right\rangle\right\rangle_{E s}\left\langle\left\langle\Psi\left|\hat{E}^{(-)}\left(t_{T}\right) \hat{E}^{(+)}\left(t_{T}\right)\right| \Psi\right\rangle\right\rangle_{E s},
\end{aligned}
$$

where $\langle\cdots\rangle_{E s}$ denotes the ensemble average over all the possible values of $\alpha_{m}\left(\omega_{m}\right)$. Here, the field operator $\hat{E}^{(+)}\left(t_{d}\right)$ at the detectors $d=C, T$ can be expressed as the sum

$$
\hat{E}^{(+)}\left(t_{d}\right)=\sum_{m} \sum_{\rightarrow=-\rightarrow, \cdots>} \hat{E}_{m \rightarrow d}^{(+)}\left(t_{d}\right)
$$

where the subfield operators

$$
\hat{E}_{m \rightarrow d}^{(+)}\left(t_{d}\right) \propto \int \mathrm{d} \omega \mathrm{e}^{-i \omega\left[t_{d}-l_{m} \rightarrow_{d} / c\right]} \hat{a}_{m}(\omega)
$$




\section{The physics of thermal light second-order interference beyond coherence}

take into account the propagation of each detected photon $\mathrm{m}$ from the point-like source where each corresponding subfields $\mathrm{m}$ is generated to the point-like detector $d=C, T$ through either the long path $l_{m^{-\rightarrow}}=L_{d}$ or the short path $l_{m \rightarrow d}=S_{d}$.

We can now describe the correlation in the intensity fluctuations in Eq. (13) in terms of the interference of all the possible "two photon-detection amplitudes"

$$
\left[\begin{array}{c}
m \rightarrow C \\
n \rightarrow T
\end{array}\right] \doteq\left\langle\alpha_{m}\left(\omega_{m}\right)\left|\hat{E}_{m \rightarrow C}^{(+)}\left(t_{C}\right)\right| \alpha_{m}\left(\omega_{m}\right)\right\rangle\left\langle\alpha_{n}\left(\omega_{n}\right)\left|\hat{E}_{n \rightarrow T}^{(+)}\left(t_{T}\right)\right| \alpha_{n}\left(\omega_{n}\right)\right\rangle,
$$

with $\rightarrow=-\rightarrow, \cdots>$, associated with all the possible contributions of two different subfields $\mathrm{m}$ and $\mathrm{n}$ to a two-photon detection, in analogy to a HBT experiment. However, since the time delay between the long (dashed red) and the short (dotted blue) paths (Fig. $2(\mathrm{a}))$ is beyond the coherence time of the source, interference can occur only if each given subfield $\mathrm{m}, \mathrm{n}$ undertakes the same type of path in the two-interfering two-photon amplitudes. There are therefore only four possible ways for these amplitudes to interfere with each other as in the expression

$$
\begin{aligned}
\left\langle\Delta I_{C}\left(t_{C}\right) \Delta I_{T}\left(t_{T}\right)\right\rangle & \propto\left\langle\sum_{m \neq n}\left[\begin{array}{c}
m \rightarrow C \\
n \rightarrow T
\end{array}\right]\left[\begin{array}{c}
m \rightarrow T \\
n \rightarrow C
\end{array}\right]^{*}+\left[\begin{array}{c}
m \cdots C \\
n \rightarrow T
\end{array}\right]\left[\begin{array}{c}
m \cdots T \\
n \rightarrow C
\end{array}\right]^{*}\right\rangle_{E s} \\
& +\left\langle\sum_{m \neq n}\left[\begin{array}{c}
m \rightarrow C \\
n \rightarrow T
\end{array}\right]\left[\begin{array}{c}
m \rightarrow T \\
n \cdots C
\end{array}\right]^{*}+\left[\begin{array}{c}
m \cdots C \\
n \rightarrow T
\end{array}\right]\left[\begin{array}{l}
m \cdots T \\
n \rightarrow C
\end{array}\right]^{*}\right\rangle_{E s}
\end{aligned}
$$

of the correlation in the intensity fluctuations, which follows from Eq. (13) by using Eqs. $(12,14,16)$. Here, the large number of contributing subfields $m, n$ spans in a continuous way all the possible eigenvalues $\alpha_{\omega}=\alpha_{m}\left(\omega_{m}\right), \alpha_{n}\left(\omega_{n}\right)$ in Eq. (16), which contribute to the ensemble average in Eq. (17) according to the probability distribution associated with the P function in Eq. (2). One can therefore write the first two contributions in Eq. (17) in terms of the phase-independent terms $\left|G^{\left(L_{C}, L_{T}\right)}\left(t_{C}, t_{T}\right)\right|^{2}$ and $\left|G^{\left(S_{C}, S_{T}\right)}\left(t_{C}, t_{T}\right)\right|^{2}$ (defined by Eqs. (11)) as

$$
\begin{aligned}
& \left\langle\sum_{m \neq n}\left[\begin{array}{l}
m \rightarrow C \\
n \rightarrow T
\end{array}\right]\left[\begin{array}{c}
m \rightarrow T \\
n-\rightarrow C
\end{array}\right]^{*}+\left[\begin{array}{c}
m \cdots C \\
n \cdots T
\end{array}\right]\left[\begin{array}{l}
m \cdots T \\
n \cdots C
\end{array}\right]^{*}\right\rangle_{E s} \\
& \simeq\left|G^{\left(L_{C}, L_{T}\right)}\left(t_{C}, t_{T}\right)\right|^{2}+\left|G^{\left(S_{C}, S_{T}\right)}\left(t_{C}, t_{T}\right)\right|^{2}=2 a^{2} \bar{r}^{2} .
\end{aligned}
$$

These interference contributions arise from the interference of two-photon amplitudes associated with two paths of the same type (long or short) within the coherence length of the source. Evidently, interference terms of this type arise in an analogous way also in standard HBT interferometers (Fig. 2(b)) where $\left(L_{C}, L_{T}\right)$ or $\left(S_{C}, S_{T}\right)$ define the path lengths from the output ports of the beam splitter to the two detectors. Since, in this case, the paths associated with the two corresponding interfering amplitudes completely overlap spatially, neither of these two terms can be sensitive to any phase delay, as expected for standard HBT interference. 


\section{The physics of thermal light second-order interference beyond coherence}

On the other hand, by again using Eqs. $(2,11,16)$, the last two interference contributions in Eq. (17) can be expressed as (c.c. stands for complex conjugate)

$$
\begin{gathered}
\left\langle\sum_{m \neq n}\left[\begin{array}{c}
m \rightarrow C \\
n \rightarrow T
\end{array}\right]\left[\begin{array}{c}
m-\rightarrow T \\
n \cdots C
\end{array}\right]^{*}+\left[\begin{array}{c}
m \cdots C \\
n-\rightarrow T
\end{array}\right]\left[\begin{array}{c}
m \cdots T \\
n \rightarrow C
\end{array}\right]^{*}\right\rangle_{E s}=\left\langle\sum_{m \neq n}\left[\begin{array}{c}
m \rightarrow C \\
n \cdots T
\end{array}\right]\left[\begin{array}{c}
m-T \\
n \rightarrow C
\end{array}\right]^{*}\right. \\
\simeq\left[G^{\left(L_{C}, L_{T}\right)}\right]^{*} G^{\left(S_{C}, S_{T}\right)}\left(t_{C}, t_{T}\right)+c . c .=2 a^{2} \bar{r}^{2} \cos \left(\varphi_{C}-\varphi_{T}\right) .
\end{gathered}
$$

Here, the two detected photons either have undertaken the $\left(L_{C}, S_{T}\right)$ path or the $\left(L_{T}, S_{C}\right)$ path, corresponding to pairs of two paths of different types (Fig. 2(c)), due to the absence of "which path information" in the propagation after the beam splitter. Therefore, these terms are sensitive to the phase difference $\varphi_{C}-\varphi_{T}$ between the two unbalanced interferometers leading to the observation of sinusoidal fringes, which are unique to this interference phenomenon with respect to standard HBT interference. This is also very different from Franson-type interference where the two photons either take the $\left(L_{C}, L_{T}\right)$ or the $\left(S_{C}, S_{T}\right)$ path, leading to a dependence on the sum $\varphi_{C}+\varphi_{T}$ of the two interferometer relative phases.

Interestingly, one can notice that it does not matter how far the long and short paths are apart from each other beyond the coherence length of the source since each subfields m,n always evolve through two possible paths of the same type (either $L=L_{C}, L_{T}$ or $S=S_{C}, S_{T}$ ) which length difference is within the coherence length of the source. Therefore the corresponding interfering two photon-amplitudes are always indistinguishable. Of course, the larger is the path unbalance in each Mach Zehnder interferometer the larger has to be the emission time delay between the photons $\mathrm{m}$ and $\mathrm{n}$ associated with the corresponding subfields triggering the two-fold detection at approximately equal detection times. However, given the continuous nature of the thermal field there is not, in principle, any constraint to such a time delay. This is evidently very different from the situation in the Franson interferometer where the time delay associated with the interferometer unbalance is required to be within the the coherence time of the pump laser used to generate the entangled pair of photons.

Remarkably, the "incoherent" sum in Eq. (17) of the two-photon interference contributions in Eqs. (18) and (19) leads to the interference of the two "effective" twopath amplitudes associated with the two pairs $\left(L_{C}, L_{T}\right)$ and $\left(S_{C}, S_{T}\right)$ of correlated paths as in Eq. (13). However, these are not interfering two-photon detection amplitudes, since no pairs of photon can have undertaken these two pairs of paths, with length difference $L-S$ beyond the coherence length of the source, in an indistinguishable manner. On the other hand, such two-path interference is a "collective" result of all the possible two-photon interference contributions in Eq. (17) as depicted in Figs. 2(b) and $2(c)$.

\section{Conclusions and perspectives}

We have described here how thermal light second-order interference beyond the coherence time arises, for any pair $(m, n)$ of detected photons, from the interference 
of two-photon detection amplitudes without contradicting our understanding of twophoton coherence.

In particular, for each pair of interfering two-photon detection amplitudes, each detected photon can undertake only paths of the same type (either L or $\mathrm{S}$ ). Indeed, only in this case the corresponding path differences $L_{C}-L_{T}$ or $S_{C},-S_{T}$ are within the coherence length of the source (Figs. 2(b) and 2(c)).

If the $m$ photon undertakes always the same type of path as the $n$ photon before triggering a two-fold detection (Fig. 2 (b)) no sinusoidal fringes could be observed. These "HBT interference contributions" are usual in standard HBT experiments (Fig. $1(\mathrm{~b}))$. However, the novelty here is that the $(\mathrm{m}, \mathrm{n})$ pair of indistinguishable photons can also undertake indistinguishable pairs of different types of paths, $\left(L_{C}, S_{T}\right)$ interfering with $\left(L_{T}, S_{C}\right)$. Remarkably, these "non-HBT interference/contributions" are sensitive to difference $\varphi_{C}-\varphi_{T}$ between the phases in the two unbalanced interferometer. Evidently, in this case, the emission time delay between the two photons is of the order of $(L-S) / c$ which is beyond the coherence time of the source. However, since the thermal field is a continuous field, there is no limit, in principle, to such a time delay and therefore to the path unbalance in each Mach-Zehnder interferometer.

Interestingly, even if the described HBT and non-HBT contributions add incoherently, an effective interference between the two pairs $\left(L_{C}, L_{T}\right)$ and $\left(S_{C}, S_{T}\right)$ of correlated paths arises from the collective contribution of all the possible pairs of photons emitted by the thermal source. This two-path interference is evidently beyond the thermal light coherence length. One can think that even if two-photon interference occurs within the coherence length of the source, a "collective" second-order interference can occur beyond such coherence length. In other words, the "incoherent" sum of all possible two-photon interference contributions can generate "coherence" in a collective manner according with the statistics of the thermal source.

We emphasize that the same physics described here naturally applies also to experiments in the spatial domain where the two Mach Zehnder interferometers in Fig. 2 (a) are substituted by two double slits [40, 41].

\section{Acknowledgments}

Research was partially sponsored by the Army Research Laboratory and was accomplished under Cooperative Agreement Number W911NF-17-2-0179. The views and conclusions contained in this document are those of the authors and should not be interpreted as representing the official policies, either expressed or implied, of the Army Research Laboratory or the U.S. Government. The U.S. Government is authorized to reproduce and distribute reprints for Government purposes notwithstanding any copyright notation herein.

V.T is also thankful to Kurt Jacobs for useful discussions.

This work is dedicated to Professor Wolfgang P. Schleich on the occasion of his 60th birthday. Indeed, the interference effect at the heart of this work was first predicted at 
the Institute of Quantum Physics in Ulm together with Johannes Seiler, who completed his bachelor thesis under my supervision. It was thanks to Wolfgang's encouragement and advice that the description of this phenomenon was finally published in New Journal of Physics as a Fast Track Communication [1]. I am extremely thankful to him for believing in me and supporting me in all my research even before I joined him in Ulm. Wolfgang has been and will always be a source of inspiration for me, not only because he is an outstanding scientist but also because he is dedicated to supporting young scientists and valuing their research. Thank you Wolfgang for your friendship and for being such a wonderful mentor for me! Happy birthday!

\section{ORCID iD}

V. Tamma https://orcid.org/0000-0002-1963-3057

\section{References}

[1] V. Tamma and J. Seiler, "Multipath correlation interference and controlled-not gate simulation with a thermal source," New J. Phys. 18, 032002 (2016).

[2] R. Hanbury Brown and R.Q. Twiss, "Correlation between photons in two coherent beams of light," Nature 177, 27 - 29 (1956).

[3] R. Hanbury Brown and R.Q. Twiss, "A test of a new type of stellar interferometer on sirius," Nature 178, 1046 -1048 (1956).

[4] W. P. Schleich, " Quantum Optics in Phase Space" (Berlin: Wiley-VCH, 2001).

[5] R.J. Glauber Quantum Theory of Optical Coherence: Selected Papers and Lectures (John Wiley and Sons, 2007).

[6] M.O. Scully, and M.S. Zubairy, Quantum Optics (Cambridge University Press, 1997).

[7] Y.H. Shih An Introduction to Quantum Optics (CRC Press Taylor and Francis group, 2011).

[8] L. Mandel and E. Wolf Optical Coherence and Quantum Optics (Cambridge University Press, 1995).

[9] R.J. Glauber, "Photon correlations," Phys. Rev. Lett. 10, 84 (1963).

[10] R.J. Glauber, "One hundred years of light quanta," Nobel Lecture 8 Dec 2005, (The Nobel Foundation 2005).

[11] C.O.Alley and Y.H.Shih Proceedings of the Second International Symposium on Foundations of Quantum Mechanics in the Light of New Technology ed of Japan P S (Tokyo, 1986), pp 47 - 52; Y. H. Shih and C. O. Alley, "New type of Einstein-Podolsky-Rosen-Bohm experiment using pairs of light quanta produced by optical parametric down conversion," Phys. Rev. Lett. 61, 2921 (1988).

[12] C. K. Hong, Z. Y. Ou, and L. Mandel, "Measurement of subpicosecond time intervals betweens two photons by interference," Phys. Rev. Lett. 59, 2044 (1987).

[13] J D Franson, "Bell inequality for position and time," Phys. Rev. Lett. 62(19) 2205-2208 (1989).

[14] T. Legero, T. Wilk, M. Hennrich, G. Rempe, and A. Kuhn, "Quantum Beat of Two Single Photons," Phys. Rev. Lett. 93, 070503 (2004).

[15] H. Kim, O. Kwon, W. Kim, and T. Kim, "Spatial two-photon interference in a Hong-Ou-Mandel interferometer," Phys. Rev. A 73, 023820 (2006).

[16] J. Liu, Y. Zhou, W. Wang, R.F. Liu, K. He, F.L. Li, and Z. Xu, "Spatial second-order interference of pseudothermal light in a Hong-Ou-Mandel interferometer," Optics Express 21(16), 19209 19218 (2013). 
The physics of thermal light second-order interference beyond coherence

[17] V. Tamma and S. Laibacher, "Multiboson correlation interferometry with multimode thermal sources," Phys. Rev. A 90, 063836 (2014).

[18] V. Tamma and S. Laibacher, "Multiboson correlation interferometry with arbitrary single-photon pure states" Phys. Rev. Lett. 114, 243601 (2015).

[19] Y. S. Ihn, Y. Kim, V. Tamma, Y.-H.Kim, "Second-Order Temporal Interference with Thermal Light: Interference beyond the Coherence Time," Phys. Rev. Lett. 119, 263603 (2017).

[20] M. Genovese "Real applications of quantum imaging," Journal of Optics 18(7), 073002 (2016).

[21] G.B. Lemos, V. Borish, G.D. Cole, S. Ramelow, R. Lapkiewicz, and A. Zeilinger, "Quantum imaging with undetected photons," Nature 512, 409-412 (2014).

[22] J. Sprigg, T. Peng, and Y.H. Shih, "Super-resolution imaging using the spatial-frequency filtered intensity fluctuation correlation," Scientific Reports 6, 38077 (2016).

[23] T.B. Pittman, Y.H. Shih, D.V. Strekalov, and A.V. Sergienko, "Optical imaging by means of two-photon quantum entanglement," Phys. Rev. A 52, R3429 (1995).

[24] A. Valencia, G. Scarcelli, M. D'Angelo, and Y.H. Shih "Two-photon imaging with thermal light," Phys. Rev. Lett. 94, 063601 (2005).

[25] M. D'Angelo and Y.H. Shih,"Quantum Imaging," Laser Phys. Lett. 2(12), 567 - 596 (2005).

[26] G. Scarcelli, V. Berardi, and Y.H. Shih, "Can two-photon correlation of chaotic light be considered as correlation of intensity fluctuations?," Phys. Rev. Lett. 96, 063602 (2006).

[27] A. Gatti, E. Brambilla, M. Bache, and L. A. Lugiato, "Correlated imaging, quantum and classical," Phys. Rev. A 70, 013802 (2004).

[28] F. Ferri, D. Magatti, A. Gatti, M. Bache, E. Brambilla, and L. A. Lugiato, "High-resolution ghost image and ghost diffraction experiments with thermal light," Phys. Rev. Lett. 94, 183602 (2005).

[29] H. Chen, T. Peng, and Y.H. Shih, " $100 \%$ correlation of chaotic thermal light," Phys. Rev. A 88, 023808 (2013).

[30] K.-H. Luo, B.-Q. Huang, W.-M. Zheng and L.-A.Wu "Nonlocal Imaging by Conditional Averaging of Random Reference Measurements," Chin. Phys. Lett. 29(7), 074216 (2012).

[31] P. Kok, "Photonic quantum information processing," Contemporary Physics 57(4), 526 - 544 (2016).

[32] M. Nielsen and I. Chuang Quantum Computation and Quantum Information (Cambridge Series on Information and the Natural Sciences, Cambridge University Press, 2000).

[33] S. Aaronson and A. Arkhipov, in Proceedings of the forty-third annual ACM symposium on Theory of computing (ACM, New York), pp. 333-342 (2011).

[34] S. Laibacher and V. Tamma, "From the physics to the computational complexity of multiboson correlation interference," Phys. Rev. Lett. 115, 243605 (2015).

[35] V. Tamma and S. Laibacher, "Multi-boson correlation sampling," Quantum Inf. Process. 15(3), $1241-1262(2016)$.

[36] V. Tamma and S. Laibacher, "Boson sampling with non-identical single photons," J. Mod. Opt. 63, $41(2015)$.

[37] V. Giovannetti, S. Lloyd, and L. Maccone, "Advances in quantum metrology," Nature Photonics $\mathbf{5}(4), 222-229$ (2011).

[38] V. Giovannetti, S. Lloyd, and L. Maccone, "Quantum-Enhanced Measurements: Beating the Standard Quantum Limit," Science 306(5700), 1330-1336 (2004).

[39] J. Dowling, "Quantum optical metrology - the lowdown on high-N00N states," Contemporary Physics 49(2), 125 - 143 (2008).

[40] M. Cassano, M. D'Angelo, A. Garuccio, T. Peng, Y.H. Shih and V. Tamma, "Spatial interference between pairs of disjoint optical paths with a single chaotic source," Opt. Express 6, 6589-6603 (2017).

[41] M. D'Angelo, A. Mazzilli, F. V. Pepe, A. Garuccio, and V. Tamma, "Characterization of two distant double-slit by chaotic light second-order interference," Scientific Reports 7, 2247 (2017).

[42] J. P. Olson, K. R. Motes, P. M. Birchall, N. M. Studer, M. LaBorde, T. Moulder, P. P. Rohde and J. P. Dowling, Phys. Rev. A 96 (2017) p. 013810. 
1

2

3

4

5

6

7

8

9

The physics of thermal light second-order interference beyond coherence

[43] O. Zimmermann and V. Tamma, "Which role does multiphoton interference play in small phase estimation in quantum Fourier transform interferometers?," Int. J. Quantum Inf. 15, 1740020 (2017).

[44] M. D'Angelo, A. Garuccio, and V. Tamma, "Toward real maximally path-entangled N -photonstate sources," Phys. Rev. A 77, 063826 (2008).

[45] Arecchi F, Gatti E and Sona A 1966 Physics Letters 2027 - 29 ISSN 0031-9163

[46] T. Peng, V. Tamma, and Y.H. Shih, "Experimental controlled-not gate simulation with, thermal light," Scientific Reports 6, 30152 (2016).

[47] S. Wölk, W. Merkel, W.P. Schleich, I.S. Averbukh, and B. Girard, "Factorization of numbers with Gauss sums: I. Mathematical background," New J. Phys. 13, 103007 (2011).

[48] V. Tamma, "Analogue algorithm for parallel factorization of an exponential number of large integers: I. theoretical description," Quantum Information Processing 15(12), 5259 - 5280 (2015).

[49] V. Tamma, "Analogue algorithm for parallel factorization of an exponential number of large integers: II. optical implementation," Quantum Information Processing 15(12), 5243 - 5257 (2015).

[50] V. Tamma, H. Zhang, X. He, A. Garuccio, W.P. Schleich, and Y.H. Shih, "Factoring numbers with a single interferogram," Phys. Rev. A 83, 020304 (2011).

[51] V. Tamma, C.O. Alley, W.P. Schleich, and Y.H. Shih, "Prime Number Decomposition, the Hyperbolic Function and Multi-Path Michelson Interferometers," Foundations of Physics 42(1), 111 - 121 (2012).

[52] E.C.G. Sudarshan, "Equivalence of semiclassical and quantum mechanical description of statistical light beams," Phys. Rev. Lett. 10, 277 (1963).

[53] Peng T, Chen H, Shih Y and Scully M O 2014 Phys. Rev. Lett. 112(18) 180401

[54] Chen H, Peng T and Shih Y 2013 Phys. Rev. A 88(2) 023808 